Tropical Journal of Pharmaceutical Research February 2019; 18 (2): 385-390

ISSN: $1596-5996$ (print); 1596-9827 (electronic)

(c) Pharmacotherapy Group, Faculty of Pharmacy, University of Benin, Benin City, 300001 Nigeria.

\title{
Delirium complications in post-general anesthesia: An observational study in China
}

\author{
Fei Fei ${ }^{1}$, Shan $\mathrm{Yu}^{2 *}$ \\ ${ }^{1}$ Department of Anesthesiology, the Affiliated Huai'an Hospital of Xuzhou Medical University, Huai'an, Jiangsu 223001, \\ ${ }^{2}$ Department of Anesthesiology, Union Hospital, Tongji Medical College, Huazhong University of Science and Technology, \\ Wuhan, Hubei 430022, China
}

*For correspondence: Email: SherryrHillsk@yahoo.com; Tel: 0086-027-84309778

Sent for review: 12 August 2018

Revised accepted: 14 January 2019

\begin{abstract}
Purpose: To evaluate emergence agitation and delirium signs in general anesthesia (GA) and postanesthesia care units (PACUs), and associated risk factors.

Methods: Adult patients $(n=380)$ exposed to GA were recruited over a period of 1 year and five months for this study, and were assessed for emergence delirium (ED) using Richmond AgitationSedation Scale (RASS). Confusion Assessment Method for Intensive Care Unit (CAM-ICU) was used to assess delirium signs on admission to, and during stay in PACU at $30 \mathrm{~min}, 1 \mathrm{~h}$, and at discharge. Signs consistent with delirium were classified as hyperactive or hypoactive based on a positive CAM-ICU assessment and RASS score. Multivariable logistic regression was used to assess potential risk factors for delirium such as age, American Society of Anesthesiologists (ASA) classification, and opioid and benzodiazepine (BZD) exposure during stay in PACU.

Results: Emergence delirium (ED) occurred in 69 (18.2\%) patients out of which 41 (59.4\%) were also CAM-ICU +ve on admission to PACU, with 22 (31.9\%), 11 (15.9\%), and $6(8.7 \%) C A M-I C U+v e$ at 30 min, $1 \mathrm{~h}$, and at discharge from PACU, respectively. Therefore, 28 (7.4\%) patients had ED with no associated signs of delirium. A total of $117(30.8 \%)$ patients had signs of delirium (CAM-ICU +ve) during admission to PACU, $58.1 \%$ of whom had hypoactive features based on their RASS scores. Signs of delirium during stay in PACU were observed in 64 (16.8\%) patients, while 61 (16.1\%), 28 (7.4 $\%)$, and 14 (3.7\%) patients were CAM-ICU +ve at $30 \mathrm{~min}, 1 \mathrm{~h}$, and at discharge from PACU, respectively. The results of multivariate logistic regression showed that total perioperative opioid administration (fentanyl equivalent) was independently associated with signs of delirium during stay in $P A C U$, after relevant covariate adjustment $(p=0.03)$. However, age, BZD exposure (midazolam equivalent), and ASA classification did not show significant association with signs of delirium during stay at PACU. The result of sensitivity analysis showed that duration of anesthesia was independently associated with signs of delirium during stay in PACU.

Conclusion: The results of this study suggest that in patients undergoing GA, signs of delirium are common in the immediate postoperative period, with incidence highest on arrival at the PACU and decreasing gradually during stay in PACU. Hypoactive features are common and more prominent during stay in PACU, when compared to hyperactive features.
\end{abstract}

Keywords: Emergence delirium, Anesthesia, Post-anesthesia care unit, Hypoactive features, Association

This is an Open Access article that uses a funding model which does not charge readers or their institutions for access and distributed under the terms of the Creative Commons Attribution License (http://creativecommons.org/licenses/by/4.0) and the Budapest Open Access Initiative (http://www.budapestopenaccessinitiative.org/read), which permit unrestricted use, distribution, and reproduction in any medium, provided the original work is properly credited.

Tropical Journal of Pharmaceutical Research is indexed by Science Citation Index (SciSearch), Scopus, International Pharmaceutical Abstract, Chemical Abstracts, Embase, Index Copernicus, EBSCO, African Index Medicus, JournalSeek, Journal Citation Reports/Science Edition, Directory of Open Access Journals (DOAJ), African Journal Online, Bioline International, Open-J-Gate and Pharmacy Abstracts 


\section{INTRODUCTION}

Delirium, also known as acute state of confusion, is a brain disorder characterized by modification of level of consciousness, disorganized thinking and inattentiveness, with signs which could be either hyperactive or hypoactive. Hyperactive signs include restlessness, rapid mood swings, and agitation, while the hypoactive signs include inattentiveness, sluggishness and lethargy. Delirium occurs in about 60 - $80 \%$ of patients under mechanical ventilation, and $20-50 \%$ of patients during their length of stay (LOS) in hospital [1-3]. Indeed, studies have shown an independent association between brain abnormality and prolonged LOS, health care cost, extended cognitive impairment and increased incidence of mortality [4-7].

Recent studies have suggested that early postoperative delirium in PACU might be associated with poor prognosis [6]. In addition, delirium occurring during stay in PACU might lead to supplementary delirium during postoperative care [7]. The diagnosis of delirium is difficult because emergence from GA usually presents with similar signs which include changes in attentiveness, disorganized thinking and altered mental status. It has been hypothesized that signs of delirium are most frequent after GA, but they diminish with time [6]. It has also been speculated that delirium signs may persist in a significant number of patients following discharge from PACU, and that hypoactive signs may be common [6]. Patientspecific or anesthetic features such as advanced age, drugs and exposure have been shown to be closely associated with increased incidence of delirium [7]. The aim of this study was to assess patients for development of ED in the PACU after exposure to $G A$, and the associated risk factors during stay in the PACU.

\section{METHODS}

\section{Patients and general information}

This cross-sectional study received approval from Institutional Review Board of the Second People's Hospital of Huai'an (approval code: RSH112015) and informed consent was acquired from participants. The International ethical guidelines for health-related research involving humans was followed [9]. Patient confidentiality was strictly maintained. Training in delirium assessment was given to four PAC nurses by the main investigator with experience in CAMICU [1] delirium assessments. Non-cardiac surgery patients in PAC admission post GA with volatile anesthetics were included. Patients were allocated by nurse in charge (round-robin method) to the PAC nurses. Therefore, that was a convenience sampling study of randomly allocated patients to four nurses. Exclusion criteria included patients with medical history of brain injuries, severe dementias, or neuromuscular disorders.

Non-cardiac surgery patients on volatile GA ( $\mathrm{n}=$ 380) were recruited over a 1-year and five months period for this study. Patients in the following categories were excluded: (1) nonChinese- speaking or deaf patients; (2) patients with history of severe dementia, anoxic brain injury or neuromuscular disorders; and (3) patients who did not sign written informed consent with their family members. The study protocol was approved by the Review Board of Union Hospital, Tongji Medical College, Huazhong University of Science and Technology, Hubei, China.

\section{Data collection}

Data collected from the patients included deidentified demographics such as age, gender, race and ASA classification; health history such as comorbid diseases, chronic alcohol or illicit drug use, smoking status, details of anesthetic course such as length of anesthetic exposure and volatile agent used, induction agent and dose. Others were lowest intraoperative vital signs such as temperature, oxygen saturation, systolic and diastolic blood pressure; perioperative medications used such as BZD, opioids and ketamine; PACU vital signs, blood product administration, Aldrete scores and verbal pain scores.

\section{Assessment and definitions}

Face valid definitions for ED and PACU delirium signs were determined based on literature review and expert opinion. Emergence delirium was defined as agitation after discontinuation of the inhaled anesthetic based on RASS score of +1 to +4. Patients were assessed for delirium signs using CAM-ICU performed at the point of admission to PACU at $30 \mathrm{~min}, 1 \mathrm{~h}$, and at discharge from the PACU. Delirium signs were deemed presentif patients were CAM-ICU positive at any time point. Hyperactive delirium signs were defined as RASS scores of +1 to +4 for agitated patients accompanied by a positive CAM-ICU. Hypoactive delirium signs were defined as RASS scores in the range of -3 to 0 for somnolent or calm patients, accompanied by a positive CAM-ICU. Postoperative delirium was defined as delirium that continued beyond the PACU or occurred in the hospital ward or ICU. 


\section{Statistical analysis}

Data are presented as median with interquartile range (IQR) and percentages where applicable. Multiple logistic regression analysis was used to determine the relationship between perioperative risk factors and signs of delirium during stay in the PACU.

\section{RESULTS}

\section{Baseline characteristics}

Table 1: Clinical and demographic characteristics

\begin{tabular}{|c|c|}
\hline Parameter & Total $(n=380)$ \\
\hline Mean age (years) & 56 \\
\hline Gender $(\mathrm{M} / \mathrm{F})^{*}$ & $210 / 170$ \\
\hline \multicolumn{2}{|l|}{ Surgery } \\
\hline General & $92(24.2 \%)$ \\
\hline Urology & $88(23.1 \%)$ \\
\hline Orthopedic & $68(17.9 \%)$ \\
\hline Neuro & $24(6.3 \%)$ \\
\hline Vascular & $18(4.7 \%)$ \\
\hline Others & $89(23.4 \%)$ \\
\hline \multicolumn{2}{|l|}{ Comorbid disorder } \\
\hline Cancer & $124(32.6 \%)$ \\
\hline Cardiovascular disorder & $62(16.3 \%)$ \\
\hline Diabetes & $52(13.7 \%)$ \\
\hline Congestive heart failure & $16(4.2 \%)$ \\
\hline Liver disorder & $10(2.6 \%)$ \\
\hline Dementia (mild) & $5(1.3 \%)$ \\
\hline ASA classification & $3(2-3)$ \\
\hline 1 & $15(3.9 \%)$ \\
\hline 2 & $172(45.2 \%)$ \\
\hline 3 & $186(48.9 \%)$ \\
\hline 4 & $10(2.6 \%)$ \\
\hline Duration of anesthesia (min) & $138(86-205)$ \\
\hline \multicolumn{2}{|l|}{ Inhalational agent } \\
\hline Sevoflurane & $198(52.1 \%)$ \\
\hline Desflurane & $142(37.4 \%)$ \\
\hline Isoflurane & $40(10.5 \%)$ \\
\hline BZDs (midazolam equivalent, mg) & $2(0-2)$ \\
\hline Opioids (fentanyl equivalent, $\mu \mathrm{g}$ ) & $380(200-550)^{* *}$ \\
\hline \multicolumn{2}{|l|}{ Verbal pain score } \\
\hline PACU admission & $0(0-3)$ \\
\hline PACU discharge & $0(0-2)$ \\
\hline \multicolumn{2}{|l|}{ Aldrete score } \\
\hline PACU admission & $8(8-8)$ \\
\hline PACU discharge & $10(9-10)$ \\
\hline
\end{tabular}

As shown in Table 1, IQR for age was 56 (43 66 years), ASA classification $3(2-3)$ and duration of anesthesia 138 (86 - $205 \mathrm{~min}$ ). About $44.7 \%$ of the patients were females, while 55.3 $\%$ were males. On admission to PACU, Aldrete scores for patients without and with delirium signs were $8(7-8)$ and $8(8-9)$, respectively, while the corresponding verbal pain scores were $0(0-2)$ and $0(0-3)$, respectively. During discharge from PACU, Aldrete scores for patients without and with delirium signs were 9 (8 - 9.8) and $10(9-10)$, respectively, while the corresponding verbal pain scores were $1(0-3.4)$ and $0(0-2)$, respectively.

\section{Emergence and PACU delirium signs}

Emergence delirium occurred in 69 (18.2\%) patients out of which $41(59.4 \%)$ were also CAM-ICU +ve on admission to PACU, with 22 (31.9\%), $11(15.9 \%)$, and $6(8.7 \%)$ continuing to be CAM-ICU +ve at $30 \mathrm{~min}, 1 \mathrm{~h}$, and discharge from PACU, respectively. Therefore, $28(7.4 \%)$ patients had ED with no associated signs of delirium. A total of 117 (30.8\%) patients had signs of delirium (CAM-ICU +ve) during admission to PACU, and $58.1 \%$ of these had hypoactive features.

Signs of delirium during stay in PACU were observed in $64(16.8 \%)$ patients, while 61 (16.1 $\%), 28(7.4 \%)$, and 14 (3.7\%) patients were CAM-ICU +ve at $30 \mathrm{~min}, 1 \mathrm{~h}$, and during discharge from PACU, respectively. In addition, $90.6 \%$ of the 64 patients who had signs of delirium amid PACU stay, had hypoactive features. These results are shown in Table 2.

Table 2: Summary of outcomes

\begin{tabular}{lc}
\hline Outcomes & $\begin{array}{c}\text { Total } \\
(\mathbf{n}=\mathbf{3 8 0})\end{array}$ \\
\hline Emergence agitation & $69(18.2 \%)$ \\
Signs of delirium at PACU & $117(30.8 \%)$ \\
admission & $68(58.1 \%)$ \\
Hypoactive & $49(41.9 \%)$ \\
Hyperactive & $64(16.8 \%)$ \\
Signs of delirium amid PACU stay & \\
Hypoactive & $58(90.6 \%)$ \\
Hyperactive & $6(9.4 \%)$ \\
CAM-ICU +ve at 30 min & $61(16.1 \%)$ \\
CAM-ICU +ve at 1 h & $28(7.4 \%)$ \\
CAM-ICU +ve at PACU discharge & $14(3.7 \%)$ \\
\hline
\end{tabular}

\section{Results of multiple regression analysis}

Results of multivariate logistic regression showed that total perioperative opioid administration (fentanyl equivalent) was independently associated with signs of delirium during stay in PACU after relevant covariate adjustment $(p=$ 0.03 ). However, age, BZD exposure (midazolam equivalent), and ASA classification did not show significant association with delirium signs amid PACU stay. Result of sensitivity analysis showed that duration of anesthesia was independently associated with signs of delirium amid PACU stay. These results are shown in Table 3. 
Table 3: Perioperative delirium signs risk-factors amid PACU stay

\begin{tabular}{ll}
\hline Variable & $\boldsymbol{P}$-value \\
\hline Age (years) & 0.23 \\
ASA classification & 0.31 \\
BZDs (midazolam equivalent, $\mathrm{mg})$ & $0.36{ }^{*}$ \\
Opioids (fentanyl equivalent, $\mu \mathrm{g})$ & $0.03^{*}$ \\
\hline${ }^{*}$ Total preoperative, intraoperative and PACU; ${ }^{\#}$ Non- \\
linear association
\end{tabular}

\section{DISCUSSION}

Emergence from anesthesia is often accompanied by signs of delirium such as fluctuating mental status and inattention. The present study assessed patients for development of ED in PACU after exposure to $G A$, and associated risk factors during stay in the PACU. The results showed that about $18.2 \%$ of patients had ED after GA, and a higher incidence of signs of delirium was observed on admission to PACU with the patients exhibiting hypoactive features. While signs of delirium were significantly diminished during stay in PACU, $3.7 \%$ of patients had ongoing delirium signs during discharge from PACU regardless of whether they met the criteria for discharge or not. This suggests that such patients might not be counted among those presenting signs of ED.

The number of vital surgeries performed globally per annum is higher than 230 million, and PACU delirium is suggestive of post-operative delirium. This means that a considerable number of patients who leave the PACU are at increased risk of delirium and delirium-related complications $[4,7]$. In this study, total perioperative opioid administration was independently associated with signs of delirium amid PACU stay after relevant covariate adjustments. However, age, BZD exposure, and ASA classification did not show significant association with signs of delirium during stay in PACU. Results of sensitivity analysis showed that duration of anesthesia was independently associated with delirium signs amid PACU stay. Studies involving the assessment of unusual emergence pattern from GA have reported ED prevalence of nearly $5 \%[14,15]$.

It is not clear if these signs on admission to PACU are indicative of ED or are simply typical signs of emergence from GA. Unfortunately, there is no typical definition of complete emergence from GA. In this study, signs of delirium were prolonged in $7.4 \%$ of patients $1 \mathrm{~h}$ after admission to PACU, and in $3.7 \%$ of patients at point of discharge from PACU. These results are in agreement with those previously reported [10]. Consideration should be centered on early detection and management of ED, since at the point of discharge from PACU, ED is usually associated with post-operative delirium and decline in cognitive function [6,7]. In addition, patients with signs of delirium at point of discharge from PACU may have a type of ongoing delirium rather than the easily reversible sedative-related delirium which may even be worse [4].

The incidence of signs of delirium observed in this study is less than the $45 \%$ reported in a previous study [13]. In the study, elderly patients were assessed for post-operative delirium following complete recovery from anesthesia, and it was observed that the patients (>70 years) were predictably at increased risk of ED. Studies have shown that CAM-ICU is more specific than sensitivity analysis when used to assess ED in less seriously ill patients, particularly during their stay in PACU $[4,13]$. Therefore, it appears that ED was under-diagnosed in this study. Although neuro-psychiatric assessment using Diagnostic and Statistical Manual of Mental Disorders (DSM) is usually considered as standard, it is capital-intensive and time-consuming [11].

In this study, CAM-ICU was used because of its simplicity and feasibility [4]. Results of this study showed that pain scores at the point of admission into and discharge from PACU were minimal and comparable clinically in patients with and without delirium, an indication that pain might be a factor in the pathogenesis of delirium. Previous studies have reported a steady relationship between opioid analgesics and perioperative delirium, with a few of them suggesting increased risk $[2,4,16]$ and some proposing no relationship [17], while others suggested a decline in incidence of delirium [5]. While it is important to control pain in perioperative procedures, it is also important to note that over-zealous drug administration may induce ED. Studies have shown that postoperative delirium is closely associated with preoperative states, degree of surgical injuries, and sedative/analgesic drug exposure [18-21].

Age has also been shown to be associated with ED [15]. In this study, patients had median age of 56 years, with a few $<40$ or $>65$ years in age, an indication that age may be a major factor in the pathogenesis of ED. In addition, $94 \%$ of patients fell under 2 or 3-ASA classification. There was no connection between BZD usage and delirium signs. These results are in agreement with those previously reported $[16,22]$. While ED might be ancillary to pain, most of the patients that had it were CAM-ICU +ve, and were on admission in the PACU. There was 
no significant difference in pain scores between patients that had signs of delirium and those that did not present the signs during admission to PACU. The patients had indications of brain disorders such as disorganized thinking or inattention, an indication that hyperactive features which occurred in a large number of the patients were not induced by pain. These results are in agreement with the reports of previous studies [23,24].

\section{Limitations of the study}

The likely limitations of this study include: (1) possibility of confounding factors in regression analysis; (2) small sample size; (3) inability to establish if patients had postoperative delirium or mild cognitive impairment before exposure to GA; (4) inability to assess pain score in patients during the emergence time, and (5) the use of a single race/population.

\section{CONCLUSION}

The results of this study suggest that in patients undergoing $\mathrm{GA}$, signs of delirium are common in the immediate postoperative period, with the incidence highest on arrival at PACU and decreasing gradually during their stay in PACU. Hypoactive features are common and more prominent during patients' stay in PACU, compared to hyperactive features.

\section{DECLARATIONS}

\section{Conflict of Interest}

No conflict of interest associated with this work.

\section{Contribution of Authors}

We declare that this work was done by the authors named in this article and all liabilities pertaining to claims relating to the content of this article will be borne by the authors. All the authors contributed equally to this work. Fei Fei performed all the experiments. Shan $\mathrm{Yu}$ designed and supervised this study.

\section{REFERENCES}

1. Ely EW, Inouye SK, Bernard GR, Gordon S, Francis J, May L, Truman B, Speroff T, Gautam S, Margolin R, et al. Delirium in mechanically ventilated patients: validity and reliability of the confusion assessment method for the intensive care unit (CAM-ICU). JAMA 2001; 286(21): 2703-2710.

2. Pandharipande $P$, Cotton BA, Shintani A, Thompson J, Pun BT, Morris JA Jr, Dittus R, Ely EW. Prevalence and risk factors for development of delirium in surgical and trauma intensive care unit patients. J Trauma 2008; 65(1): 34-41.

3. Ouimet $S$, Kavanagh BP, Gottfried SB, Skrobik $Y$. Incidence, risk factors and consequences of ICU delirium. Intensive Care Med 2007; 33(1): 66-73.

4. Card E, Pandharipande $P$, Tomes $C$, Lee C, Wood J, Nelson D, Graves A, Shintani A, Ely EW, Hughes $C$. Emergence from general anaesthesia and evolution of delirium signs in the post-anaesthesia care unit. $\mathrm{Br} J$ Anaesth 2015; 115(3): 411-417.

5. Morrison RS, Magaziner J, Gilbert M, Koval KJ, McLaughlin MA, Orosz G, Strauss $E$, Siu $A L$. Relationship between pain and opioid analgesics on the development of delirium following hip fracture. $J$ Gerontol A Biol Sci Med Sci 2003; 58(1): 76-81.

6. Neufeld KJ, Leoutsakos JM, Sieber FE, Pauldine RW, Gerold KB, Hang J, Smith TH. Outcomes of early delirium diagnosis after general anesthesia in the elderly. Anesth Analg 2013; 117(2): 471-478.

7. Sharma PT, Sieber FE, Zakriya KJ, et al. Recovery room delirium predicts postoperative delirium after hip-fracture repair. Anesth Analg 2005; 101(4): 1215-1220.

8. Sessler CN, Gosnell MS, Grap MJ, Brophy GM, O'Neal $P V$, Keane KA, Tesoro EP, Elswick RK. The Richmond Agitation-Sedation Scale: validity and reliability in adult intensive care unit patients. Am J Respir Crit Care Med 2002; 166(10): 1338-1344.

9. International Ethical Guidelines for Health-related Research Involving Humans, Fourth Edition. Geneva. Council for International Organizations of Medical Sciences (CIOMS); 2016.

10. Aldrete JA. Modifications to the postanesthesia score for use in ambulatory surgery. J Perianesth Nurs 1998; 13(3): 148-155.

11. American Psychiatric Association. Diagnostic and Statistical Manual of Mental Disorders, 4th Edn, text revision. Washington, DC. American Psychiatric Association, 2000.

12. Gusmao-Flores D, Figueira Salluh Jl, Chalhub RA, Quarantini LC. The confusion assessment method for the intensive care unit (CAM-ICU) and intensive care delirium screening checklist (ICDSC) for the diagnosis of delirium: a systematic review and meta-analysis of clinical studies. Crit Care 2012; 16(4): R115.

13. Neufeld KJ, Leoutsakos JS, Sieber FE, Joshi D, Wanamaker BL, Rios-Robles J, Needham DM. Evaluation of two delirium screening tools for detecting post-operative delirium in the elderly. $\mathrm{Br} J$ Anaesth 2013; 111(4): 612-618.

14. Lepouse $C$, Lautner CA, Liu L, Gomis $P$, Leon $A$. Emergence delirium in adults in the post-anaesthesia care unit. Br J Anaesth 2006; 96(6): 747-753.

15. Radtke FM, Franck $M$, Hagemann $L$, Seeling $M$, Wernecke KD, Spies CD. Risk factors for inadequate emergence after anesthesia: emergence delirium and hypoactive emergence. Minerva Anestesiol 2010; 76(6): 394-403.

Trop J Pharm Res, February 2019; 18(2): 389 
16. Marcantonio ER, Juarez G, Goldman L, Mangione CM, Ludwig LE, Lind L, Katz N, Cook EF, Orav EJ, Lee TH. The relationship of postoperative delirium with psychoactive medications. JAMA 1994; 272(19): 15181522.

17. Sieber FE, Mears S, Lee H, Gottschalk A. Postoperative opioid consumption and its relationship to cognitive function in older adults with hip fracture. J Am Geriatr Soc 2011; 59(12):2256-2262.

18. Ansaloni L, Catena F, Chattat R, Fortuna D, Franceschi $C$, Mascitti P, Melotti RM. Risk factors and incidence of postoperative delirium in elderly patients after elective and emergency surgery. Br J Surg 2010; 97(2): 273280.

19. Koebrugge B, van Wensen RJ, Bosscha K, Dautzenberg $P L$, Koning $\mathrm{OH}$. Delirium after emergency/elective open and endovascular aortoiliac surgery at a surgical ward with a high standard delirium care protocol. Vascular 2010; 18(5): 279-287.
20. Salata K, Katznelson R, Scott BW, Carroll J, Lindsay TF, Djaiani $G$. Endovascular versus open approach to aortic aneurysm repair surgery: rates of postoperative delirium. Can J Anaesth 2012; 59(6): 556-561.

21. Vasilevskis EE, Han JH, Hughes CG, Ely EW. Epidemiology and risk factors for delirium across hospital settings. Best Pract Res Clin Anaesthesiol 2012; 26(3): 277-287.

22. Taipale $P G$, Ratner $P A$, Galdas $P M$, Jillings $C$, Manning $D$, Fernandes $C$, Gallaher J. The association between nurse-administered midazolam following cardiac surgery and incident delirium: An observational study. Int $J$ Nurs Stud 2012; 49(9): 1064-1073.

23. Chester JG, Beth HM, Rudolph JL. Serial administration of a modified Richmond Agitation and Sedation Scale for delirium screening. J Hosp Med 2012; 7(5): 450-453.

24. Tieges Z, McGrath A, Hall RJ, MacLullich AM. Abnormal level of arousal as a predictor of delirium and inattention: an exploratory study. Am J Geriatr Psychiatry 2013; 21(12): 1244-1253. 